\title{
Unravelling the Mechanism of Pressure Induced Amorphization of Phase Change Materials
}

\author{
S. Caravati, ${ }^{1,2}$ M. Bernasconi, ${ }^{1, *}$ T. D. Kühne, ${ }^{2}$ M. Krack, ${ }^{3}$ and M. Parrinello ${ }^{2}$ \\ ${ }^{1}$ Dipartimento di Scienza dei Materiali, Università di Milano-Bicocca, Via R. Cozzi 53, I-20125, Milano, Italy \\ ${ }^{2}$ Department of Chemistry and Applied Biosciences, ETH Zurich, USI Campus, Via Giuseppe Buffi 13, 6900 Lugano, Switzerland \\ ${ }^{3}$ Paul Scherrer Institut, 5232 Villigen, Switzerland \\ (Received 11 August 2008; published 19 May 2009)
}

\begin{abstract}
Based on ab initio molecular dynamics simulations, we identify the atomistic mechanism of the pressure induced amorphization of $\mathrm{Ge}_{2} \mathrm{Sb}_{2} \mathrm{Te}_{5}$. The simulations reveal that homopolar $\mathrm{Ge} / \mathrm{Sb}$ bonds appear in cubic $\mathrm{Ge}_{2} \mathrm{Sb}_{2} \mathrm{Te}_{5}$ under pressure, giving rise to square rings rotated by $45^{\circ}$ with respect to the crystalline axis whose formation is induced by the displacement of Te atoms filling the voids of neighboring $\mathrm{Ge} / \mathrm{Sb}$ stoichiometric vacancies. The concentration of these topological defects increases with pressure up to $21 \mathrm{GPa}$ at which the system is destabilized and transforms into an amorphous phase in agreement with experiments.
\end{abstract}

Chalcogenide alloys are the subject of extensive experimental and theoretical research because of their use in optical [digital versatile disk (DVD)] and electronic [phase change memories (PCM)] devices. Both applications rely on the fast and reversible phase change between the crystalline and amorphous phases induced by heating either via laser irradiation (DVD) or the Joule effect (PCM) [1]. Although a great deal of progress has been made in the past few years in the understanding of the basic properties of this class of materials [1-7], the microscopic mechanism of the fast phase change still deserves further investigation.

Useful information on the mechanism of the phase transformation can be gained by inducing the transition via means other than increasing temperature.

Recently, it is has been shown experimentally that cubic crystalline $\mathrm{Ge}_{2} \mathrm{Sb}_{2} \mathrm{Te}_{5}$ (GST), the prototypical material in this class, turns into an amorphous phase when submitted to a pressure of $20 \mathrm{GPa}$ at room temperature (RT) and for higher temperatures up to $150^{\circ} \mathrm{C}$ [8-10]. The amorphous phase can be recovered upon decompression at normal conditions if the temperature is kept below $110^{\circ} \mathrm{C}$, while at $145^{\circ} \mathrm{C}$ (just below the crystallization temperature at normal pressure) the initial fcc crystal is recovered [9]. Extended x-ray absorption fine structure (EXAFS) and $\mathrm{x}$-ray absorption near-edge structure (XANES) data on pressure amorphized GST are very similar to those of the amorphous phase generated by quenching from the melt [11]. Kolobov et al. [11] interpreted EXAFS/XANES data by assuming that in amorphous GST ( $a$-GST) Ge atoms are tetrahedrally coordinated as opposed to their octahedral coordination in the crystalline phases (hexagonal and metastable cubic). Conversion from octahedral to tetrahedral geometry would then have to be expected in pressure induced amorphization as well. Other cubic crystals along the pseudobinary lines $(\mathrm{GeTe})_{x}\left(\mathrm{Sb}_{2} \mathrm{Te}_{3}\right)_{y}$ similarly undergo amorphization above a threshold pressure which, however, depends on the concentration of vacancies on the
$\mathrm{Ge} / \mathrm{Sb}$ sublattice of the cubic rocksalt phase [10]. The higher the concentration of vacancies, the lower the transition pressure. At the same time, the hexagonal phase of GST (hex-GST), in which atoms are still octahedrally coordinated but with no vacancies, as well as the binary GeTe compound (with no vacancies) remain crystalline up to the highest pressure ( $\sim 25 \mathrm{GPa})$ investigated experimentally [10].

To shed light onto the role of vacancies in the amorphization process, we performed density-functional based simulations of GST at high pressure. Molecular dynamics simulations were performed using the scheme of Kühne et al. [12], implemented in the CP2K suite of programs [13]. We use the Perdew-Becke-Ernzerhof (PBE) [14] exchange-correlation functional and Goedecker-type pseudopotentials $[15,16]$. The Kohn-Sham orbitals were expanded in a triple-zeta valence plus polarization (triple-ZVP) Gaussian-type basis set, and the charge density was expanded in a plane wave basis set with a cutoff of 100 Ry within the QUICKSTEP scheme [17]. Brillouin zone (BZ) integration was restricted to the supercell $\Gamma$ point. A time step of 2 fs was used.

In a previous work [5], we generated a 270-atom model of $a$-GST by quenching from the melt within the same computational framework. In our $a$-GST model [5], most of the $\mathrm{Ge}$ and $\mathrm{Sb}$ atoms are 4-coordinated, while $\mathrm{Te}$ is mostly 3-coordinated in defective octahedral-like sites which recall the local environment of cubic crystalline GST ( $c$-GST). However, as much as one-third of the Ge atoms are in a tetrahedral geometry as suggested by EXAFS/XANES measurements [11]. This configuration is absent in the crystalline phase and favored in $a$-GST by the presence of homopolar (Ge-Ge and $\mathrm{Ge}-\mathrm{Sb}$ ) bonds. Similar results on the structural properties of $a$-GST have been found independently by other groups $[6,7]$.

We simulated the pressure induced amorphization of a 270-atom supercell of $c$-GST, where Te occupies one 
sublattice of the rocksalt crystal, and $\mathrm{Ge}, \mathrm{Sb}$, and vacancies are randomly placed in the other sublattice, initially at the experimental density at normal conditions. We submitted the model to increasing pressure by reducing isotropically the volume in 12 steps up to the pressure of $31 \mathrm{GPa}$, each simulation at fixed volume lasting on average $8 \mathrm{ps}$. The ion temperature was set at RT by a Langevin thermostat.

In Fig. 1, we report the evolution of the intensity of the main peaks in the structure factor $S(Q)$ which correspond to the order parameters of the crystalline phase. The full structure factor $S(Q)$ as a function of pressure is given in the additional material [18]. Crystalline order clearly disappears above $21 \mathrm{GPa}$ in agreement with experiments [8]. The $\langle 200\rangle$ peak which gives a measure of the alignment of the square rings along $\langle 100\rangle$ directions is the last to disappear.

By progressively increasing the volume of the supercell (in 12 steps lasting 7 ps each), the amorphous phase was recovered at normal conditions. Aiming at speeding up the transformation and at inducing a further decrease in the intensity of the $\langle 200\rangle$ peak, we also performed a second simulation starting from the configuration at $21 \mathrm{GPa}$. Namely, we increased temperature to $430{ }^{\circ} \mathrm{C}$ (still below the melting point) for $20 \mathrm{ps}$, and then we kept increasing pressure up to $31 \mathrm{GPa}$ at $130^{\circ} \mathrm{C}$. The model was then decompressed down to zero pressure at $130{ }^{\circ} \mathrm{C}$. The structure of the two amorphous models generated under pressure is actually very similar, the hotter model being slightly more disordered as inferred by the intensity of the $\langle 200\rangle$ peak. The transformation is irreversible because of kinetic hindrance at low temperature (below $145^{\circ} \mathrm{C}$ ) as demonstrated experimentally in Ref. [9]. The equations of state

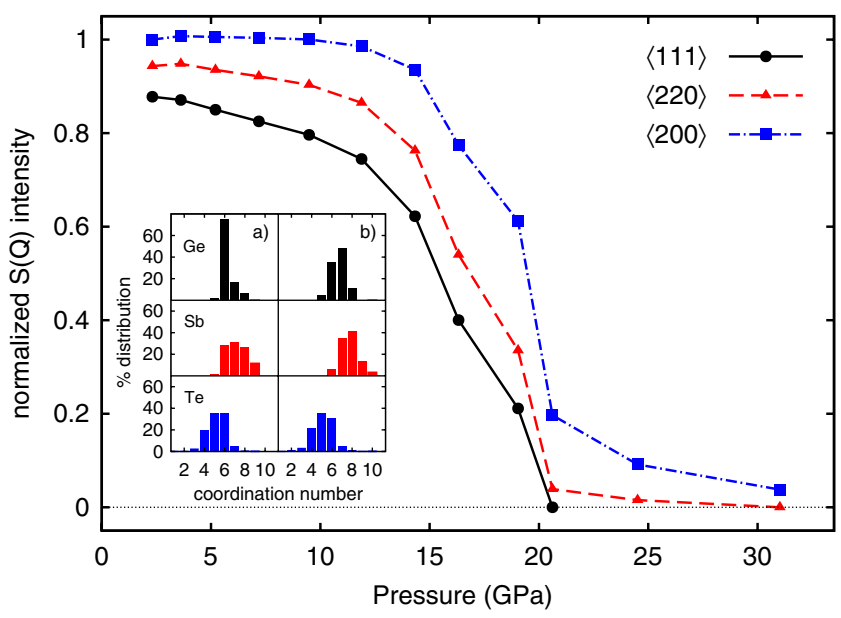

FIG. 1 (color online). Evolution with pressure of the intensity of the main peaks of the structure factor $S(Q)$ normalized to the intensities of the $\langle 200\rangle$ peak at zero pressure. Inset: Distribution of the coordination number for (a) $c$-GST and (b) $a$-GST at the same density which corresponds to a pressure of $21 \mathrm{GPa}$ in $a$-GST. The average coordination numbers are $n_{\mathrm{Ge}}=6.28$, $n_{\mathrm{Sb}}=7.18$, and $n_{\mathrm{Te}}=5.23$ for $c$-GST and $n_{\mathrm{Ge}}=6.67, n_{\mathrm{Sb}}=$ 7.72 , and $n_{\mathrm{Te}}=5.14$ for $a$-GST (at $21 \mathrm{GPa}$ ).
(EOS) of pressure amorphized GST, $c$-GST, meltquenched $a$-GST [5], and hex-GST are compared in Fig. 2. Pressure versus volumes curves for $c$-GST and pressure amorphized $a$-GST are given in Fig. S2 as additional materials [18]. In $a$-GST at high pressure, atoms are clearly more coordinated than in the crystal at the same density as shown by the distribution of coordination numbers of $c$-GST and $a$-GST (inset in Fig. 1). However, upon decompression, the amorphous phase reaches an equilibrium density slightly higher than that of our melt-quenched $a$-GST model [5] but still lower than that of $c$-GST. Namely, $a$-GST models obtained upon decompression or by quenching from the melt are, respectively, $6.5 \%$ or $8.2 \%$ less dense than $c$-GST (in reasonable agreement with the experimental density increase of $6.8 \%$ upon crystallization of melt-quenched $a$-GST [19]). We cannot assess whether this small change in the equilibrium density of $a$-GST is a distinctive property of the generation protocol (pressure versus quenching from the melt) or it is an artifact of the short span of the simulation which leads the system facing difficulties to reach a fully relaxed structure in the decompression run. Regardless of the small change in the equilibrium density, the structure of two amorphous phases (pressure-generated and melt-quenched) recovered at normal conditions turns out to be very similar. The fraction of homopolar $\mathrm{Ge} / \mathrm{Sb}$ and $\mathrm{Te} / \mathrm{Te}$ bonds is the same in both systems, and most of $\mathrm{Sb}$ and $\mathrm{Ge}$ are 4-coordinated in defective octahedral sites, while a fraction of Ge atoms are in tetrahedral geometry [5]. As in melt-quenched $a$-GST, pressure amorphized GST does not contain long chains of Te, but only dimers and trimers [5]. Partial pair correlation functions, the bond angle distribution function, and the distribution of coordination numbers are given in Figs. S3 and S4 as additional materials [18] and can be compared with the corresponding data of melt-quenched

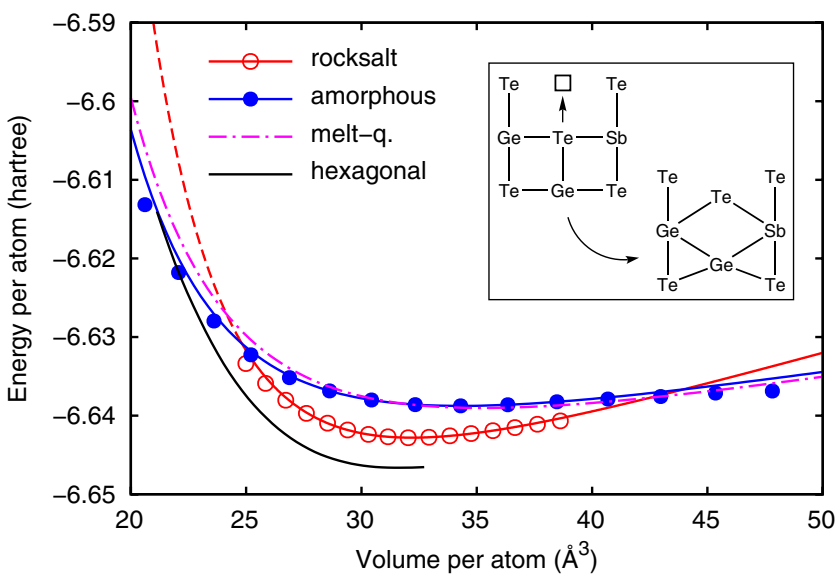

FIG. 2 (color online). Equation of state of $c$-GST, hex-GST, pressure amorphized GST (filled dots), and melt-quenched $a$-GST [5]. Energy-volume points are fitted with a Murnaghan function. Inset: Sketch of the displacement of Te atom toward the vacancy giving rise to a rotated square ring with homopolar bonds. 
$a$-GST of Ref. [5]. The distribution of the local order parameter $q$ introduced in Ref. [5] to quantify the number of tetrahedral $\mathrm{Ge}$ is also reported as additional material (Fig. S5 in [18]). The fraction of tetrahedral Ge atoms is $17 \%$, a value lower than that found in melt-quenched $a$-GST (33\%) [5] which might be a consequence of the higher equilibrium density of pressure amorphized GST with respect to melt-quenched $a$-GST. Actually, the conversion from octahedral to tetrahedral coordination of $\mathrm{Ge}$ takes place during decompression, since the most probable coordination number of Ge is as large as 6 at $21 \mathrm{GPa}$ (cf. Fig. 1). Therefore, we can exclude that the amorphization under pressure is driven by the flipping model proposed in Ref. [11] with a movement of the Ge atoms from octahedral to tetrahedral sites upon compression. This suggests that also the crystal-to-amorphous phase change exploited in the devices might be more complex than the simple model of flipping Ge.

By inspecting the atomic trajectories, we confirm instead a crucial role of the vacancies in the process. In $c$-GST, the system can increase the density upon compression without reducing too much the nearest neighbor distances by filling the vacancy voids. This is achieved by a displacement of Te atoms toward the nearest vacancy as sketched in the inset in Fig. 2. The movement of Te atom induces the breaking of the Te- $X$ bond opposite to the vacancy and the formation of new homopolar $\mathrm{Ge} / \mathrm{Sb}$ bonds. This process gives rise to squared rings rotated by $45^{\circ}$ with respect to crystal axes (inset in Fig. 2 and Fig. 3). The evolution as a function of pressure of the concentration of homopolar bonds is reported in Fig. 4. As additional material, we also report the evolution as a function of

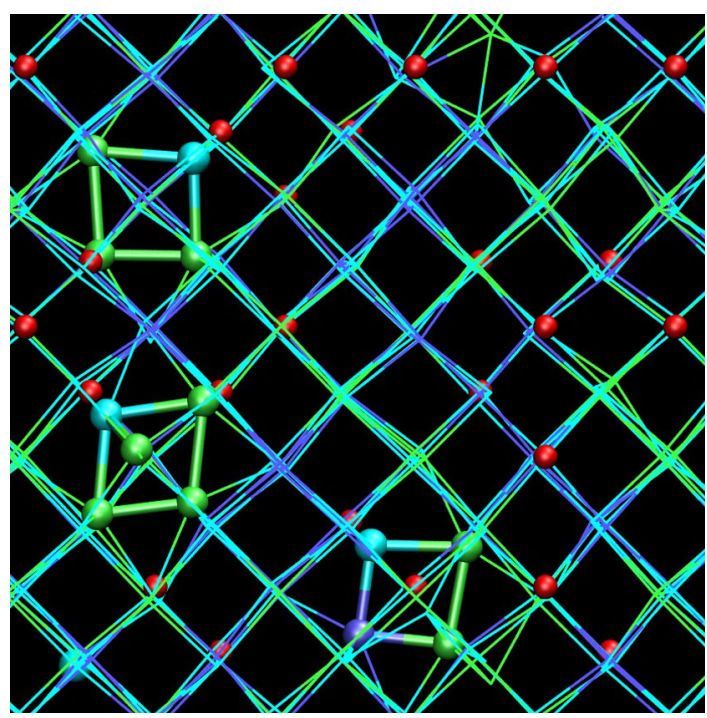

FIG. 3 (color online). Snapshot of the simulation at $14 \mathrm{GPa}$ showing the appearance of squared rings formed by homopolar $\mathrm{Ge} / \mathrm{Sb}$ bonds rotated by $45^{\circ}$ with respect to the crystal axis. Large spheres indicate atoms involved in the formation of rotated rings (blue: Ge; green: Sb; light blue: Te). Small red spheres indicate the position of $\mathrm{Ge} / \mathrm{Sb}$ vacancies. pressure of the volume occupied by vacancies defined according to Ref. [6] (Fig. S6 in Ref. [18]) and of the concentration of different types of squared rings (Fig. S7 in Ref. [18]) including the $A A A T$ Te ring $(A=\mathrm{Ge} / \mathrm{Sb})$ generated in the transformation sketched in Fig. 2 (inset). By raising the pressure, the volume assigned to vacancies decreases while the concentration of AAATe rings increases. By inspection in the electronic density of states as a function of pressure (Fig. S8 in Ref. [18]), one observes a closing of the band gap at high pressure which widens again upon decompression. The electronic density of states of $a$-GST recovered at zero pressure is similar to that of melt-quenched $a$-GST but for the presence of a higher concentration of defects states in the gap band presumably due to a too short simulation time (cf. Fig. S8 in Ref. [18]).

We speculate that the role of vacancies in the amorphization process is twofold. They increase the compressibility of the crystal at intermediate pressure and allow reaching a higher density at lower pressure via formation of rotated rings (the EOS of $c$-GST deviates from the dashed line in Fig. 2 due to the transformation connected with the formation of rotated rings). Presumably, the higher the concentration of vacancies, the higher the concentration of rotated rings. However, this does not imply that vacancies can reduce the compressibility at zero pressure as well, where rotated rings cannot form. Second, rotated rings formed thanks to vacancies destabilize the system once their concentration becomes too high. The formation of rotated rings can actually accommodate the density increase without compressing the Te- $X$ bonds, but only up to a certain extent. When pressure is too high, Te- $X$ bonds become too compressed and the system transforms into an amorphous phase. The amorphization transition is indeed driven by a higher compressibility at high pressure

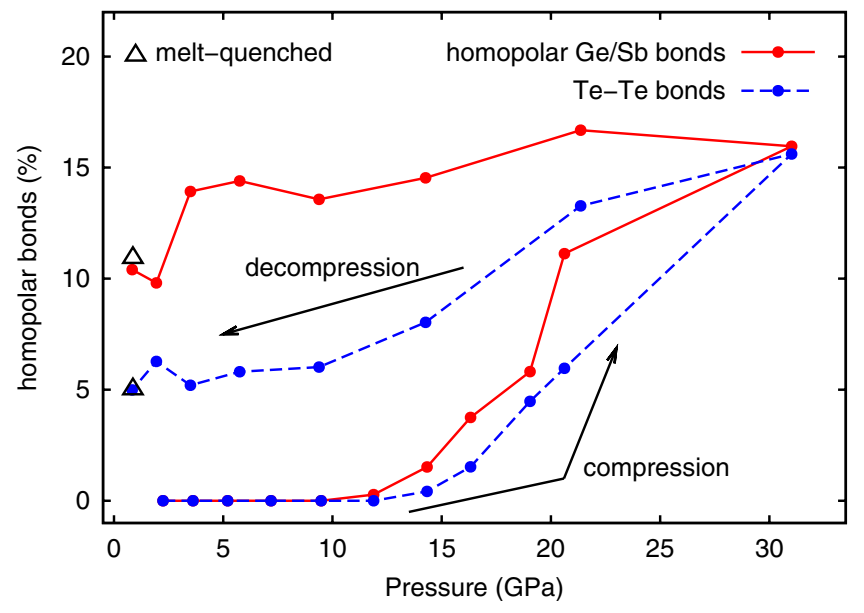

FIG. 4 (color online). Evolution with pressure of the fraction (over the total number of bonds) of $\mathrm{Ge} / \mathrm{Sb}$ and $\mathrm{Te} / \mathrm{Te}$ homopolar bonds during the compression and decompression runs. The corresponding values in melt-quenched $a$-GST [5] are given for sake of comparison. 
of $a$-GST with respect to $c$-GST as already suggested in Ref. [10]. From the EOS of Fig. 2 one recognizes that the transformation is thermodynamically favored because of the lower enthalpy of $a$-GST with respect $c$-GST. At a given (high) pressure, $a$-GST is actually denser than the crystal in spite of the fact that the opposite is true at low (zero) pressure. Although at normal conditions the average coordination numbers in $a$-GST are lower than in the crystal $(\mathrm{Ge} / \mathrm{Sb}$ are mainly 4-coordinated in $a$-GST but 6 coordinated in the crystal at normal conditions), at high pressure the amorphous phase can accommodate the increased density via an increase in coordination numbers with a lower compression of $\mathrm{Te}-X$ bonds than occurring in the crystal. On the contrary, the EOS of hex-GST [20] is always below those of $c$-GST and $a$-GST in agreement with the stability of hex-GST at high pressure revealed experimentally [10] (cf. Fig. 2). Actually, c-GST turns into $a$-GST at high pressure because the transition to hex-GST is kinetically hindered (below $250{ }^{\circ} \mathrm{C}$ at normal pressure).

Moreover, $a$-GST is more compressible than $c$-GST also at zero pressure. The bulk modulus $(B)$ at the equilibrium volume of the EOS in Fig. 2 is $11 \mathrm{GPa}$ for $a$-GST (both models) and $27 \mathrm{GPa}$ for $c$-GST [21]. For the sake of comparison with the Brillouin scattering data of Ref. [23], we also computed the bulk modulus of $\mathrm{GeSb}_{2} \mathrm{Te}_{4}$ (189-atom supercell) which turned out to be $30 \mathrm{GPa}$, equal to the experimental data within the experimental uncertainties [23,24]. According to the data of Ref. [23], also $\mathrm{GeSb}_{2} \mathrm{Te}_{4}$ is more compressible in the amorphous $(B=17.9 \pm 2.9 \mathrm{GPa})$ than in the crystalline $(B=27.3 \pm 3.7 \mathrm{GPa})$ phase, although to a smaller extent than our prediction for GST [24]. We remark that the analysis of the EOS under pressure in Refs. [9,11] yielded $B=41 \pm 2 \mathrm{GPa}$ for both crystalline GST and $\mathrm{GeSb}_{2} \mathrm{Te}_{4}$. The reason for the discrepancy with our results and with the Brillouin scattering data deserves further investigation, although we cannot exclude that it might be partially due to inaccuracies of the exchange and correlation energy functional we have adopted [25].

In summary, ab initio molecular dynamics simulations have revealed the microscopic mechanism behind the pressure induced amorphization of GST. By pressurizing the cubic crystalline phase, first Te atoms move to fill the vacancy voids giving rise to topological defects in the rocksalt crystal made of homopolar $\mathrm{Ge} / \mathrm{Sb}$ bonds in squared rings rotated by $45^{\circ}$ with respect to the crystalline axis. By further increasing pressure, the concentration of the latter topological defects increases up to $21 \mathrm{GPa}$ at which the system transforms into an amorphous phase. The amorphization occurs on the time scale of a few picoseconds, and it is driven by the higher compressibility of the amorphous phase with respect to the crystal.

We thankfully acknowledge the computational resources provided by the Barcelona Supercomputing Center-
Centro Nacional de Supercomputación, by the DEISA Consortium under project PHASEMAT, and by CSCS (Manno, CH). S. C. acknowledges support through SNSF (No. 200021-119882).

*marco.bernasconi@mater.unimib.it

[1] M. Wuttig and N. Yamada, Nature Mater. 6, 824 (2007).

[2] W. Wełnic and M. Wuttig, Mater. Today 11, 20 (2008).

[3] D. Lencer et al., Nature Mater. 7, 972 (2008).

[4] K. Shportko et al., Nature Mater. 7, 653 (2008).

[5] S. Caravati et al., Appl. Phys. Lett. 91, 171906 (2007).

[6] J. Akola and R. O. Jones, Phys. Rev. B 76, 235201 (2007).

[7] J. Hegedüs and S. R. Elliott, Nature Mater. 7, 399 (2008).

[8] A. V. Kolobov et al., Phys. Rev. Lett. 97, 035701 (2006).

[9] M. Krbal et al., Appl. Phys. Lett. 93, 031918 (2008).

[10] A. V. Kolobov et al., Appl. Phys. Lett. 91, 021911 (2007).

[11] A. V. Kolobov et al., Nature Mater. 3, 703 (2004).

[12] T. D. Kühne et al., Phys. Rev. Lett. 98, 066401 (2007).

[13] M. Krack and M. Parrinello, in High Performance Computing in Chemistry, edited by J. Grotendorst (NIC, Jülich, 2004), Vol. 25, pp. 29-51; http://cp2k.berlios.de.

[14] J. P. Perdew et al., Phys. Rev. Lett. 77, 3865 (1996).

[15] S. Goedecker et al., Phys. Rev. B 54, 1703 (1996).

[16] M. Krack, Theor. Chem. Acc. 114, 145 (2005).

[17] J. VandeVondele et al., Comput. Phys. Commun. 167, 103 (2005).

[18] See EPAPS Document No. E-PRLTAO-102-006923 for properties of crystalline and amorphous GST as a function of pressure. For more information on EPAPS, see http:// www.aip.org/pubservs/epaps.html.

[19] W. Njoroge et al., J. Vac. Sci. Technol. A 20, 230 (2002).

[20] The EOS of hex-GST was computed for the structure proposed by Kooi et al. [J. Appl. Phys. 92, 3584 (2002)] with a 270 -atom supercell.

[21] We checked that the bulk moduli do not change (within $1 \mathrm{GPa}$ ) by improving the basis set (quadruple-ZVP or plane waves [22]) or the pseudopotentials (including semicore states for $\mathrm{Ge}$ or $\mathrm{Te}$ in the valence). For crystals, improving BZ integration over a $2 \times 2 \times 2$ mesh [22] produces instead a decrease of the bulk modulus with respect to $\mathrm{CP} 2 \mathrm{~K}$ results ( $\Gamma$ point) by $3 \mathrm{GPa}$ for GST and by $7 \mathrm{GPa}$ for $\mathrm{GeSb}_{2} \mathrm{Te}_{4}$ by computing the energies of the geometries optimized with the $\mathrm{CP} 2 \mathrm{~K}$ code ( $\Gamma$ point).

[22] These calculations were performed with plane waves by using the code ABINIT (http://www.abinit.org).

[23] T. Blachowicz et al., J. Appl. Phys. 102, 093519 (2007).

[24] The values of the bulk moduli inferred from Brillouin scattering data quoted in the text are obtained by using the elastic constants in Table I and Eq. (3) of Ref. [23] as the bulk moduli reported in the last column of the same table are misprinted [M. Beghi (private communication)].

[25] For trigonal GeTe our calculated bulk modulus (PBE) at convergence in BZ integration is $36 \mathrm{GPa}$ [22], while a calculation within the local density approximation gives a value of $44.80 \mathrm{GPa}$ closer to the experimental value of $49.96 \pm 3.2 \mathrm{GPa}[26]$.

[26] R. Shaltaf et al., Phys. Rev. B 78, 205203 (2008). 